Check for updates

Cite this: RSC Adv., 2019, 9, 27674

\title{
Ce-doped UiO-67 nanocrystals with improved adsorption property for removal of organic dyes
}

\author{
Xue Dong, ${ }^{\text {ab }}$ Yongcen Lin, ${ }^{\text {ab }}$ Yuqin Ma (D) ${ }^{b}$ and Lang Zhao (D) *a
}

In this study, we report the synthesis of Ce-doped UiO-67 nanocrystals via one-step hydrothermal method and their potential use for waste water treatment to remove organic dyes. The as-prepared samples were characterized by using SEM, TEM, FT-IR, XRD, XPS and TG techniques and the results demonstrate the formation of the framework structure of the Ce-doped UiO-67. The adsorption study of the material shows that the Ce-doped $\mathrm{UiO}-67$ nanoparticles preferentially adsorb the cationic dyes such as rhodamine $\mathrm{B}$ and malachite green rather than the anionic dyes such as methyl orange. Adsorption capacities are 754.4, 589.2 and $191.6 \mathrm{mg} \mathrm{g}^{-1}$ for rhodamine B, malachite green and methyl orange respectively. Based on its zeta potential and adsorption isotherm, it is understood that Ce doping increases its electrostatic interactions, and as a consequence, improves the adsorption capacity for cationic dyes. In addition, it is found that a pseudo-second-order kinetics and the Langmuir isothermal model were suitable for describing the adsorption behavior of cationic dyes onto the Ce-doped UiO-67.

Received 19th July 2019

Accepted 22nd August 2019

DOI: 10.1039/c9ra05586j

rsc.li/rsc-advances

stability, none secondary pollution after its structure break-down ${ }^{24,25}$

\section{Introduction}

Aggravated by population, industrialization and urbanization, water pollution becomes a serious issue which needs to be resolved. Organic dyes are widely employed in the textile, paper, leather, and printing industries ${ }^{\mathbf{1}}$ which cause water coloring upon their discharge. Just a very low concentration can damage the environment and poses serious threats to human health due to their toxicity, potential mutagenicity and carcinogenicity. ${ }^{2}$ Currently, many processes such as oxidation, ${ }^{3}$ adsorption, ${ }^{4}$ coagulation/flocculation, ${ }^{5}$ photolytic degradation, ${ }^{6}$ electrocatalytic degradation, ${ }^{7}$ biological treatment, ${ }^{8}$ reverse osmosis and membrane filtration ${ }^{9-12}$ have been attempted to remove those polluting organic dyes from waste water. Among these, adsorption is considered to be a favorable approach due to its feasibility, efficiency, no by-products and easy operation. However, finding a new adsorbent material with high performance and low cost, which is easy to make remains an actual challenge. MOFs which are organic-inorganic hybrid solids with regular channels or pore structures consisting of metal ions/ clusters and organic linkers, ${ }^{\mathbf{1 3 - 1 6}}$ have received great attention in various fields including adsorption and storage, ${ }^{17}$ drug delivery, ${ }^{18}$ heterogeneous catalysis, ${ }^{19}$ sensors,${ }^{20}$ pollutant elimination, ${ }^{21}$ and energy storage and conversion. ${ }^{22}$ Among various MOFs structures, the zirconium-based MOFs of UiO series become very promising materials due to their excellent thermal, aqueous, and acid stabilities. $^{23}$ Its exceptional biocompatibility, hydrothermal and thermal

${ }^{a}$ State Key Laboratory of Rare Earth Resource Utilization, Changchun Institute of Applied Chemistry, Chinese Academy of Sciences, Changchun 130022, P. R. China. E-mail: Zhaolang@ciac.ac.cn; Fax: +86-431-85262878

${ }^{b}$ School of Chemistry and Environmental Engineering, Changchun University of Science and Technology, Changchun 130012, P. R. China and excellent chemical stability render the UiO-67 structure one of the most popular Zr-based frameworks for waste water treatment. Recently, metal ion doping of MOF-5 with Ni(II) ions exhibits enhancement in its hydrostability. ${ }^{26}$ Linnan Li et al. ${ }^{27}$ reported that a lanthanide functionalized MOF can be used as a turn-off luminescent sensory material for detection of $\mathrm{Fe}^{3+}$ in aqueous media. Jiao Cao et $a .^{28}$ reported that Co-doped MOF expands its adsorptive ability and enhances its photocatalytic performance. Xuan Yang et $a l^{29}$ reported that doping Ce(III) to MIL-96 increases its adsorption capacity for fluoride ions. However, most of the synthetic methods are cumbersome and tedious and all the reported MOF-drived materials are not scalable. ${ }^{30-33}$ Therefore, a simple and easy scalable preparation process is needed.

Herein, we report a one-step hydrothermal synthesis for doping metal ions into MOF structures. We chose Ce and UiO67 because, to the best of our knowledge, doping of Ce to UiO-67 was rarely investigated. The so obtained Ce-doped UiO-67 was then characterized by using the SEM, TEM, FT-IR, XRD, XPS and TG techniques. For waste water treatment experiments, we chose two cationic dyes, rhodamine $\mathrm{B}(\mathrm{RhB})$ and malachite green (MG), and one anionic dye, methyl orange (MO) as model compounds. The effect of initial solution $\mathrm{pH}$ on the dye adsorption was also investigated.

\section{Experimental}

\subsection{Sample preparation}

Materials and chemicals: $\mathrm{ZrCl}_{4}$ (98\%), biphenyl-4,4'-dicarboxylic acid $\left(\mathrm{H}_{2} \mathrm{BPDC}, 98 \%\right)$ and rhodmine $\mathrm{B}(\mathrm{RhB})$ were purchased from Shanghai Macklin Biochemical Co., Ltd. $\mathrm{Ce}\left(\mathrm{NO}_{3}\right)_{3} \cdot 6 \mathrm{H}_{2} \mathrm{O}$ 
(98\%) was purchased from Sinopharm Chemical Reagent Co, Ltd. Acetic acid (HAc, 99.5\%) and $N, N$-dimethylformamide (DMF, 99.5\%) were obtained from Beijing Chemical Works. All reagents and solvents were analytical grade and used directly without any further purification.

\subsection{Synthesis of Ce-doped UiO-67}

Typically, $\mathrm{ZrCl}_{4}(46.6 \mathrm{mg}), \mathrm{Ce}\left(\mathrm{NO}_{3}\right)_{3} \cdot 6 \mathrm{H}_{2} \mathrm{O}(86.8 \mathrm{mg}), \mathrm{H}_{2} \mathrm{BPDC}$ $(96.8 \mathrm{mg})$, and HAc $(0.38 \mathrm{~mL})$ were mixed and stirred for dissolution in $15 \mathrm{~mL}$ DMF at room temperature. The obtained mixture was transferred into a $25 \mathrm{~mL}$ Teflon-lined autoclave and heated to $120^{\circ} \mathrm{C}$ and maintained at this temperature for $24 \mathrm{~h}$. Subsequently the autoclave was taken out of the oven and cool down to room temperature. The solid product was isolated by centrifugation, washed with DMF three times and dried in vacuum at $100{ }^{\circ} \mathrm{C}$ overnight.

\subsection{Characterization}

The morphology of the samples was examined by using scanning electron microscopy (SEM, Hitachi S4800) with an accelerating voltage of $10 \mathrm{kV}$. The transmission electron microscopy (TEM) images were recorded on a JEOL JEM-2010EX transmission electron microscope with an accelerating voltage of 200 $\mathrm{kV}$. The Fourier transform infrared (FT-IR) spectra were collected within the $400-4000 \mathrm{~cm}^{-1}$ spectral range on a Thermo Fisher Nicolet-6700 spectrometer. The X-ray powder diffraction (XRD) patterns from $5^{\circ}$ to $80^{\circ}$ were taken on a D8 Focus diffractometer (Bruker) with $\mathrm{Cu}$ target $(40 \mathrm{kV}, 40 \mathrm{~mA})$. The X-ray photoelectron spectra (XPS) were obtained on an AXIS Ultra instrument from Kratos Analytical. The nitrogen adsorptiondesorption isotherms were collected using a Micromeritics ASAP 2020 instrument. The specific surface areas were calculated using the Brunauer-Emmett-Teller (BET) method, and the pore size distributions were evaluated using the BarrettJoyner-Halenda (BJH) model. The thermogravimetric analyses (TGA) were carried out using a TG/SDTQ600 instrument in atmosphere within temperature range of $0-900 \mathrm{~K}$. The ultraviolet-visible (UV-vis) spectroscopy measurements were carried out with a UV-3600 UV-vis spectrophotometer (Shimadzu).

\subsection{Adsorption experiments}

Adsorption experiments of the two cationic dyes (RhB and $\mathrm{MG}$ ) were conducted to evaluate the adsorption properties of the material and to understand the factors which influence the adsorption. All of the adsorption experiments were done at ambient temperature. The effect of the initial solution $\mathrm{pH}$ on the adsorption of $\mathrm{RhB}$ and $\mathrm{MG}$ by the material was examined by adjusting the solution $\mathrm{pH}$ with negligible amounts of $0.1 \mathrm{M} \mathrm{HCl}$ or $0.1 \mathrm{M} \mathrm{NaOH}$ in the range of 3-10. After adsorption, the adsorbent was separated by centrifugation at $10000 \mathrm{rpm}$ for $5 \mathrm{~min}$, and the supernatant was examined with a UV-3600 spectrophotometer at the maximum absorption wavelengths of the dyes. For adsorption kinetic studies, the experiments were performed using different adsorbent dosage $\left(1200 \mathrm{mg} \mathrm{L}^{-1}\right.$ for $\mathrm{RhB}$ and
$800 \mathrm{mg} \mathrm{L}^{-1}$ for $\mathrm{MG}$ ) at a constant temperature. The remaining concentrations of dyes in the solution were analyzed at different time intervals. The adsorption isotherms were obtained via altering the initial dye concentrations.

The adsorption capacity $\left(Q_{\mathrm{e}}, \mathrm{mg} \mathrm{g}^{-1}\right)$ was calculated according to the following eqn (1)

$$
Q_{\mathrm{e}}=\left(C_{0}-C_{\mathrm{e}}\right) V / m
$$

where $C_{0}$ and $C_{\mathrm{e}}\left(\mathrm{mg} \mathrm{L}^{-1}\right)$ are the initial and equilibrium concentrations of the dye in the solution, $V(\mathrm{~L})$ is the volume of solution, and $m(\mathrm{~g})$ is the mass of adsorbent. $Q_{\mathrm{e}}\left(\mathrm{mg} \mathrm{g}^{-1}\right)$ is the adsorption capacity of the adsorbent.

\section{Results and discussion}

\subsection{Synthesis and characterization}

The synthesis was carried out as described in the experimental section. Fig. 1(a) and (c) show TEM and SEM pictures of the as-synthesized UiO-67. The UiO-67 crystals have regular octahedron shape with an average crystal size of $400 \mathrm{~nm}$, while the Ce-doped UiO-67 crystals have the same morphology but smaller (average diameter, $200 \mathrm{~nm}$, see Fig. 1(b) and (d)). The SEM images of the Ce-doped UiO-67 crystals were in line with the EDS mappings for $\mathrm{C}$, Zr, and Ce (Fig. 2). The Ce and $\mathrm{Zr}$ mappings match the $\mathrm{C}$ mapping, indicating that Ce is well dispersed in the Ce-doped UiO-67 framework.

The FT-IR spectra of the samples are presented in Fig. 3(a). For the Ce-doped UiO-67, the characteristic peaks at 668 and $768 \mathrm{~cm}^{-1}$ are the longitudinal and transverse vibrations of the $\mathrm{Zr}-\mathrm{O}$ bonds ${ }^{34,35}$ and the peaks at $1400-1600 \mathrm{~cm}^{-1}$ (labeled with purple stars) are corresponding to the stretching of $\mathrm{O}-\mathrm{C}=\mathrm{O}$ groups. ${ }^{36}$ The peak at $1658 \mathrm{~cm}^{-1}$ is wider compared to that of the UiO-67, indicating that the Ce ions in the material are coordinating with the carbonyl group $(\mathrm{C}=\mathrm{O})$ of the $\mathrm{BDC}$ ligand. Additional a tiny bump in the peak at $1488 \mathrm{~cm}^{-1}$ is also seen due to the $\mathrm{Ce}-\mathrm{O}$ stretching and $\mathrm{Ce}-\mathrm{O}-\mathrm{C}$ vibrations in the $\mathrm{MOF},{ }^{37,38}$ demonstrating that Ce substitution to $\mathrm{Zr}$ takes place in the Ce-doped UiO-67.

The crystalline structures and phase composition of UiO67 and Ce-doped UiO-67 were characterized by XRD. As shown in Fig. $3(\mathrm{~b})$, the diffraction peaks at $5.6^{\circ}, 6.5^{\circ}, 11.2^{\circ}$ and $17.2^{\circ}$ are indexed to the typical crystalline structure of the Ce-doped UiO-67, which are similar to the characteristic peaks of the crystalline UiO-67..$^{35,39}$ When Ce is incorporated into the UiO-67 structure by replacement of $\mathrm{Zr}$, the position of the main diffraction peaks is not changed compared to that of the UiO-67, but the intensity of the diffraction peaks is increased due to the higher scattering power of Ce compared to $\mathrm{Zr}$. The incorporation of Ce influences the crystal growth of the UiO-67 units to make the crystal size of Ce-doped UiO-67 smaller, as shown in the pictures above (Fig. 1(b) and (d)). The valence state of materials was characterized using X-ray photoelectron spectroscopy (XPS) (Fig. 4). From the survey spectrum in Fig. 4(a), the elements 

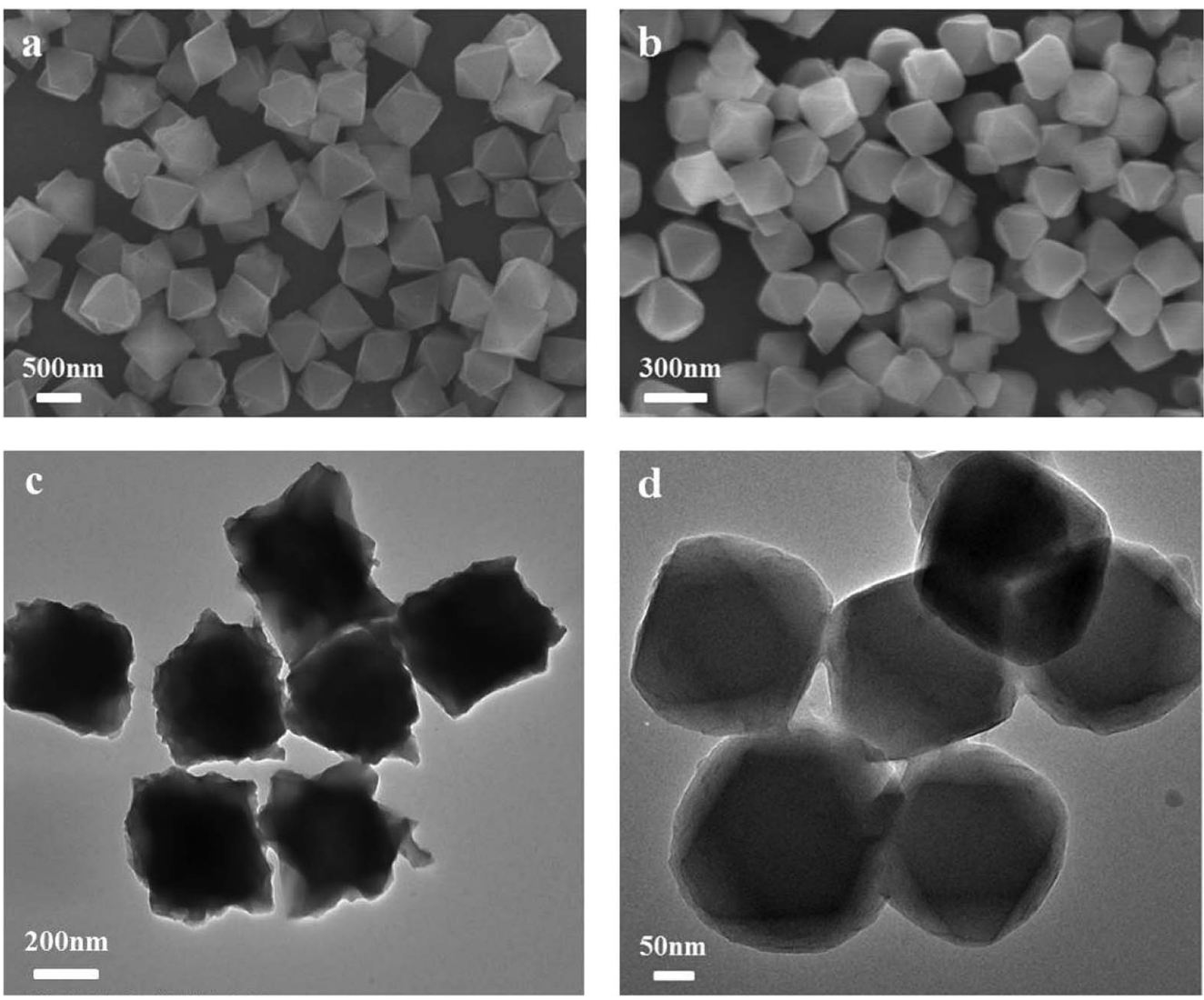

Fig. 1 SEM images of (a) UiO-67, (b) Ce-doped UiO-67; TEM images of (c) UiO-67, (d) Ce-doped UiO-67.

$\mathrm{C}, \mathrm{O}$ and $\mathrm{Zr}$ exist in the Ce-doped UiO-67 spectrum with strong intensities while the intensity of the elements $\mathrm{Ce}$ is weak, indicating that its content is low on the surface. The content of cerium in the Ce-UiO- 67 is $0.8 \%$ by the ICP analysis. Fig. 4(b) displayed the $\mathrm{C} 1 \mathrm{~s}$ spectra of Ce-doped UiO-67, the most prominent peak at $284.7 \mathrm{eV}$ is attributed to the $\mathrm{sp}^{2}$-hybridized carbon $(\mathrm{C}=\mathrm{C})$ on the benzene ring. The peak at $288.6 \mathrm{eV}$ is assigned to $\mathrm{HO}-\mathrm{C}=\mathrm{O}$ groups of the BPDC linkers. The $\mathrm{O}$ 1s spectra can be decomposed into three peaks in Fig. 4(c). ${ }^{40}$ The binding energy at $533 \mathrm{eV}$ is assigned to the $\mathrm{CO}$ groups and the peaks at $533.6 \mathrm{eV}$ correspond to the oxygen of $\mathrm{H}_{2} \mathrm{O}$. The peaks at $531.7 \mathrm{eV}$ are attributed to the $\mathrm{CeO}$ and $\mathrm{ZrO}^{37}$ It is declared that cerium can be also a metallic center binding with $\mathrm{H}_{2}$ BPDC. As shown in Fig. 4(d), the two peaks at 182.5 and $184.9 \mathrm{eV}$ in the $\mathrm{Zr} 3 \mathrm{~d}$ spectrum can be ascribed to the $\mathrm{Zr}-\mathrm{O}$ bonds. ${ }^{41}$ The Ce $3 \mathrm{~d}$ XPS spectrum could not be devoted anymore, owing to the little amount which it can be seen from EDS results (Fig. 2). All of these results further confirm that the Cedoped UiO-67 was prepared successfully. The $\mathrm{N}_{2}$ adsorption-desorption isotherms and pore size distribution are shown in Fig. 5(a) and the textural parameters are listed in Table 1. The adsorption-desorption isotherms exhibit type-I isotherm implying that Ce-doped UiO-67 is a typical microporous material. Ce-doped UiO-67 shows a BET specific surface area of $1795 \mathrm{~m}^{2} \mathrm{~g}^{-1}$, which exceeds that of pure UiO-67 nanocrystals $\left(1653 \mathrm{~m}^{2} \mathrm{~g}^{-1}\right)$. The average pore width of the channels of the Ce-doped UiO-67 $(2.047 \mathrm{~nm})$ is similar to that of UiO-67 $(2.053 \mathrm{~nm})$, while the pore volume of the Ce-doped UiO-67 $\left(0.878 \mathrm{~cm}^{3} \mathrm{~g}^{-1}\right)$ is higher than that of
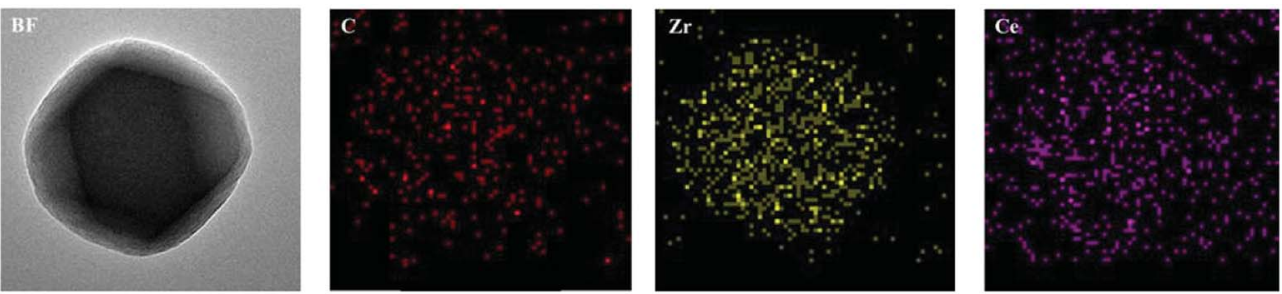

Fig. 2 EDS elemental mapping of Ce-doped UiO-67. 

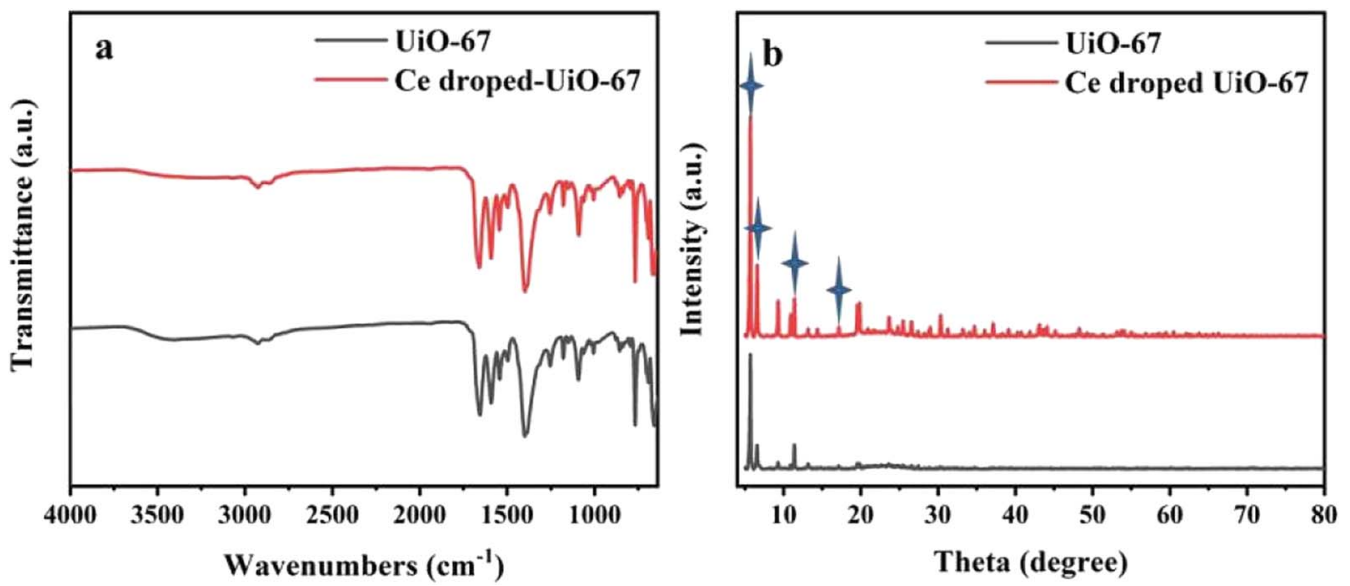

Fig. 3 (a) The FT-IR spectra of UiO-67 and Ce-doped UiO-67. (b) XRD patterns of UiO-67 and Ce-doped UiO-67.

the UiO-67 $\left(0.793 \mathrm{~cm}^{3} \mathrm{~g}^{-1}\right)$, indicating that cerium doping resulted in increased pore volume. The Ce-doped UiO-67 is expected to have better adsorption capacity compared to its corresponding parts. Fig. 5(b) gives TGA plots of weight loss of the UiO-67 and Ce-doped UiO-67 in air atmosphere. Two main weight loss steps are observed in the temperature range $0-900{ }^{\circ} \mathrm{C}$. The first weight loss in the temperature range of $25-200{ }^{\circ} \mathrm{C}$ is due to the loss of the guest molecules
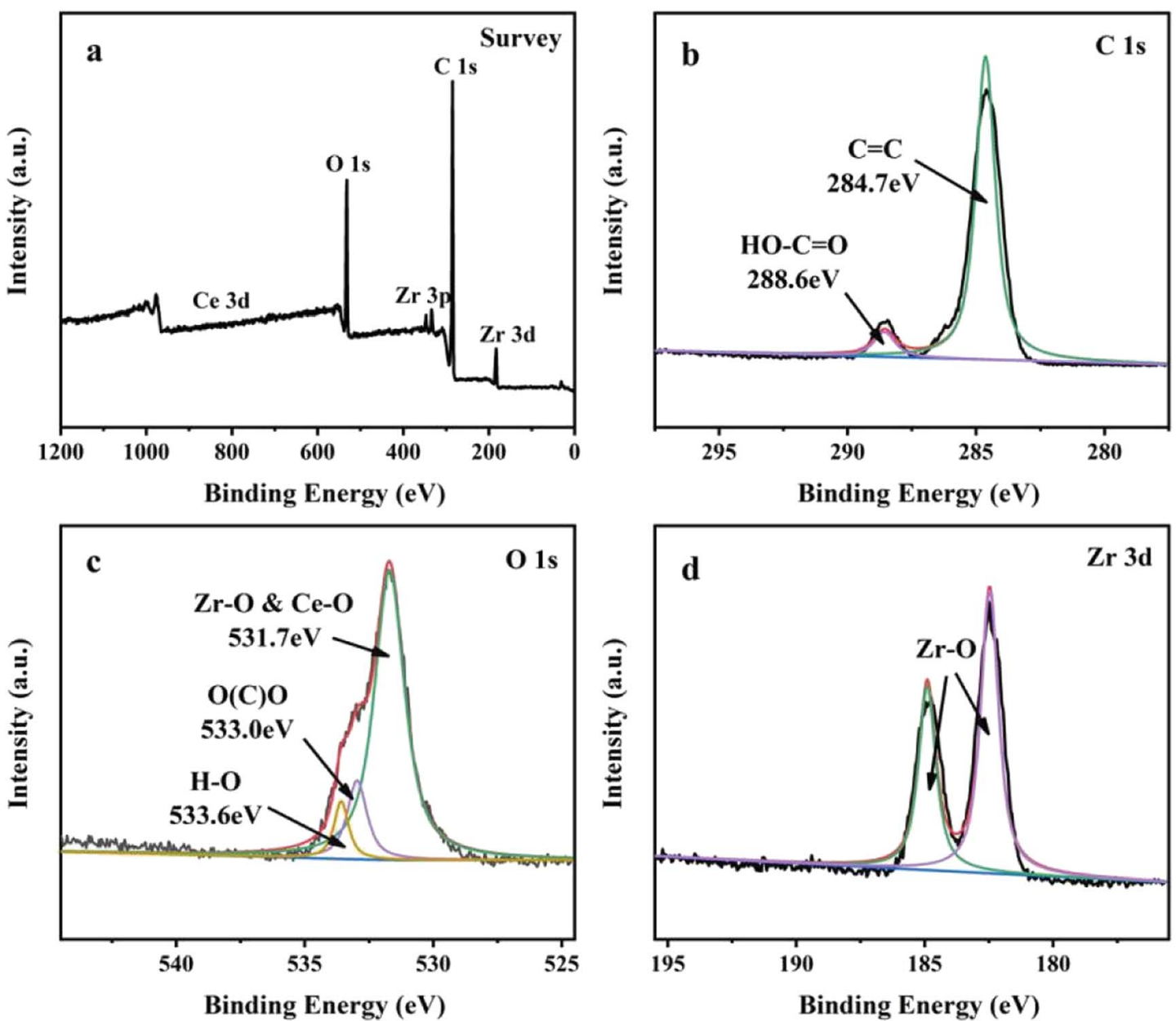

Fig. 4 XPS survey spectrum of (a) Ce-doped UiO-67, high resolution XPS spectra of (b) C 1s (c) O 1s and (d) Zr 3d obtained from C Ce-doped UiO-67. 

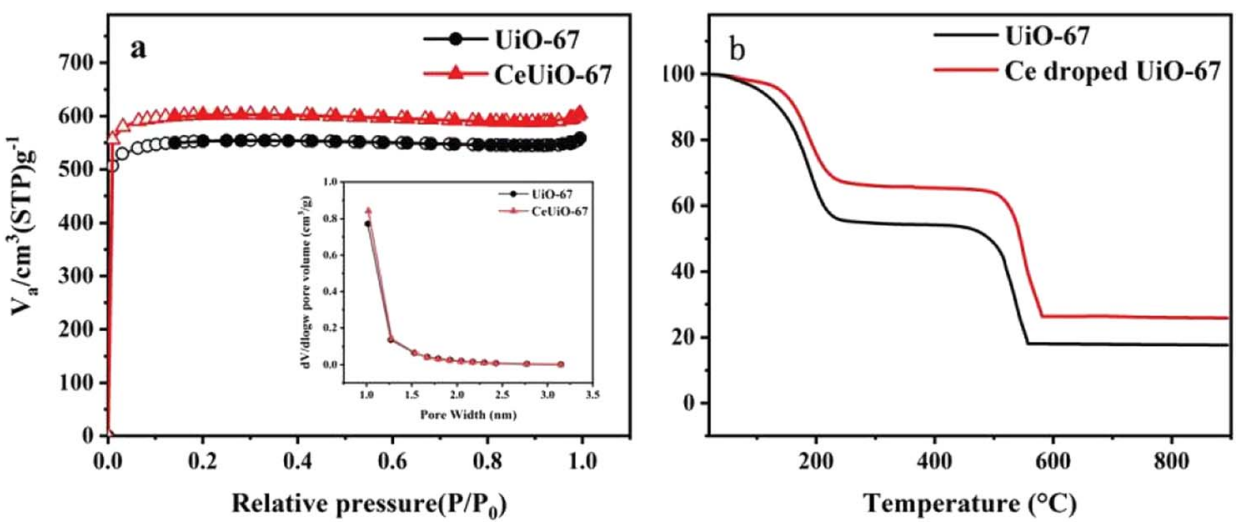

Fig. 5 (a) $\mathrm{N}_{2}$ adsorption isotherms of $\mathrm{UiO}-67$ and Ce-doped $\mathrm{UiO}-67$. The inset shows the $\mathrm{BJH}$ pore size distribution curve. (b) TGA of UiO-67 and Ce-doped UiO-67 under air atmosphere.

Table 1 Surface area and porosity characteristics of the samples

\begin{tabular}{llll}
\hline Sample & $S_{\text {BET }}\left(\mathrm{m}^{2} \mathrm{~g}^{-1}\right)$ & $d_{\text {pore }}(\mathrm{nm})$ & $V_{\text {pore }}\left(\mathrm{cm}^{3} \mathrm{~g}^{-1}\right)$ \\
\hline UiO-67 & 1653 & 2.054 & 0.793 \\
Ce-doped UiO-67 & 1795 & 2.047 & 0.878
\end{tabular}

incorporated during the synthesis. The second weight loss in the temperature range of $400-600{ }^{\circ} \mathrm{C}$ is attributed to the decomposition of the framework. The weight losses for both materials occur at the same temperature, indicating that the thermal stability of the framework is not affected by doping. For the first step, this difference was attributed to the asprepared Ce-doped UiO-67 having a smaller pore size and volume than UiO-67, which resulted in less guest solvent molecules in the former framework. For the second step, the structure is destroyed and left behind is $\mathrm{ZrO}_{2}$ for UiO-67 and $\mathrm{ZrO}_{2} / \mathrm{CeO}_{2}$ for the Ce-doped UiO-67. The remaining weight is $17 \mathrm{wt} \%$ and $26 \mathrm{wt} \%$, respectively. Thus, the TGA results further confirm that element cerium is incorporated in the framework with no much influence on its framework thermal stability. ${ }^{42-44}$

\subsection{Dye adsorption performance}

3.2.1. Adsorption kinetics. During the adsorption process, the contact time is a crucial factor, which influences the adsorption capacities of the adsorbents. As shown in (Fig. 6(b)), the adsorption of MG on the Ce-doped UiO-67 reached equilibrium within $24 \mathrm{~min}$. Similar adsorption kinetics was observed for that of RhB (Fig. 6(a)). To better understand the absorption mechanism, pseudo-first-order and pseudo-secondorder kinetic models were tried to describe the adsorption data of RhB and MG. The commonly known pseudo-first-order model (eqn (2)) and pseudo-second-order model (eqn (3)) are expressed in a linear form as follows:

$$
\begin{gathered}
\ln \left(Q_{\mathrm{e}}-Q_{t}\right)=\ln Q_{\mathrm{e}}-K_{1} t \\
t / Q_{t}=1 /\left(K_{2} Q_{\mathrm{e}}{ }^{2}\right)+t / Q_{\mathrm{e}}
\end{gathered}
$$

where $Q_{\mathrm{e}}$ and $Q_{t}\left(\mathrm{mg} \mathrm{g}^{-1}\right)$ are the capacity of dye adsorbed at equilibrium and at time $t ; K_{1}\left(\mathrm{~min}^{-1}\right)$ and $K_{2}\left(\mathrm{~g} \mathrm{~m}^{-1} \mathrm{~min}^{-1}\right)$ denote kinetic velocity constants of the pseudo-first-order and pseudo-second-order, respectively.

The fitting using the two models is given in Fig. 6 and the relevant parameters are listed in Table 2 . The correlation coefficients $R^{2}$ of pseudo-second-order kinetic model were all higher than 0.999 and obviously higher than the pseudofirst-order kinetic model for RhB and MG, implying the pseudo-second-order model is suitable to describe the adsorption behavior of Ce-doped UiO-67. The calculated $Q_{\mathrm{e}}$ value with the pseudo-second-order kinetic model was in accordance to the experimental data.

3.2.2. Adsorption isotherms. To further understand how RhB and MG are adsorbed onto Ce-doped UiO-67, the adsorption isotherms of RhB and MG with various initial concentrations were measured. As seen in Fig. 7, the adsorption of both dyes on the Ce-doped UiO-67 initially increases linearly with the increase of initial dye concentration and eventually reaches a plateau.

Moreover, the Ce-doped UiO-67 exhibited adsorption capacities of RhB (754.4 $\mathrm{mg} \mathrm{g}^{-1}$ ) and MG (589.2 $\mathrm{mg} \mathrm{g}^{-1}$ ) respectively, being significantly higher than those previously reported for UiO-67, RhB $\left(41.3 \mathrm{mg} \mathrm{g}^{-1}\right)$ and MG $\left(357.3 \mathrm{mg} \mathrm{g}^{-1}\right)^{35}$ or other porous materials (Table 3). Compared to the pristine UiO-67, Ce-doped UiO-67 featured a significantly decreased positive surface, which resulted in decreased electrostatic repulsion between the adsorbent and cationic dye molecules, facilitating their adsorption.

Subsequently, the equilibrium adsorption data were analyzed by two kinetic models, Langmuir and Freundlich isothermal models. The Langmuir adsorption isotherm represents monolayer adsorption onto a finite number of homogeneous sites, and the Freundlich adsorption isotherm describes adsorption of adsorbates onto a heterogeneous surface with different functional groups or adsorbent-adsorbate interactions.

The Langmuir isotherm is expressed as follows:

$$
C_{\mathrm{e}} / Q_{\mathrm{e}}=C_{\mathrm{e}} / Q_{\mathrm{m}}+1 /\left(K_{\mathrm{L}} Q_{\mathrm{m}}\right)
$$



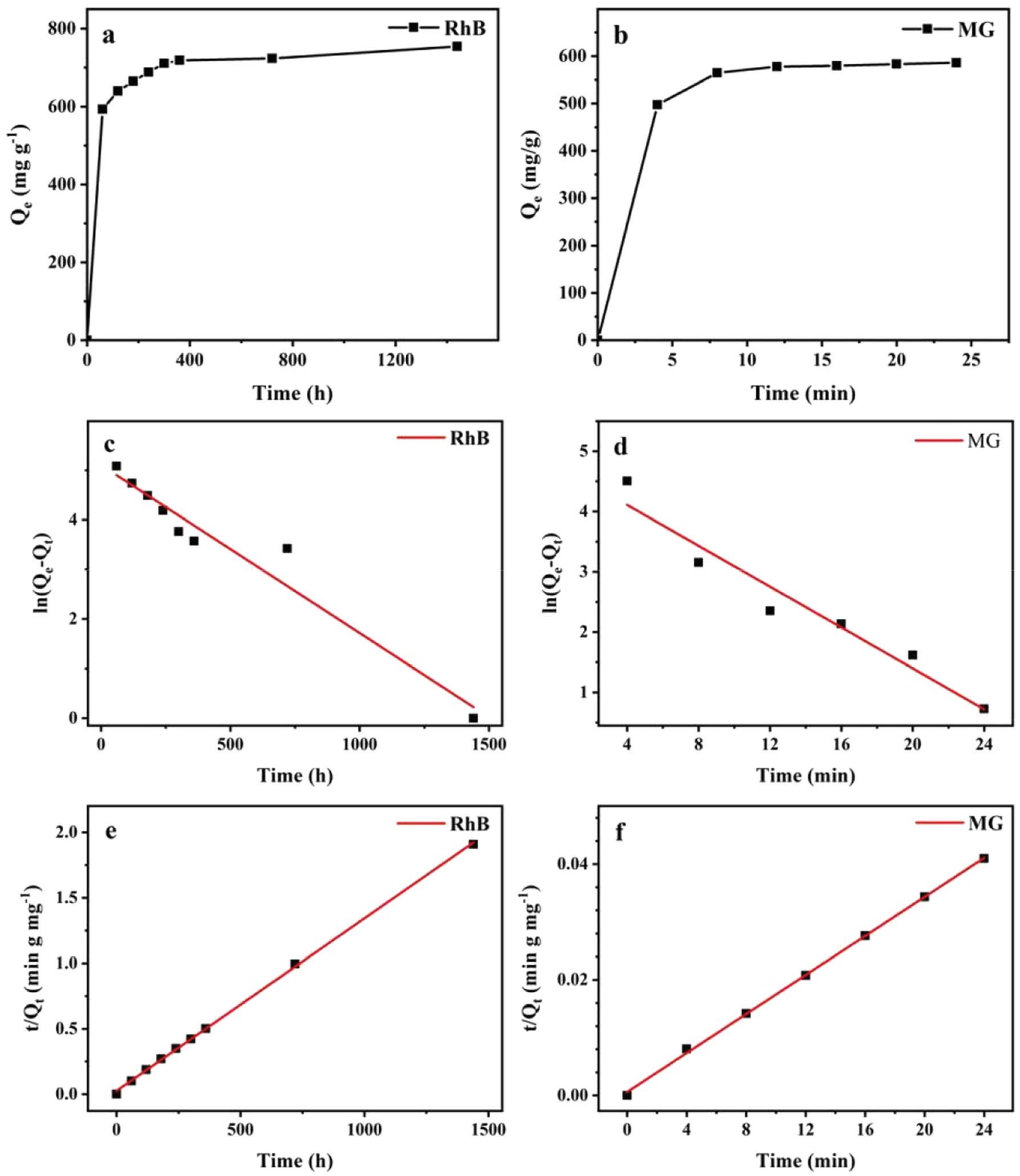

Fig. 6 Adsorption kinetics of RhB and MG onto Ce-doped UiO-67 (a and b) contact time curve plots; (c and d) pseudo-first-order and; (e and f) pseudo-second-order kinetic models (adsorption conditions: $m=5 \mathrm{mg} ; V=5 \mathrm{~mL}$ ).

where $Q_{\mathrm{e}}\left(\mathrm{mg} \mathrm{g}^{-1}\right)$ and $C_{\mathrm{e}}\left(\mathrm{mg} \mathrm{L}^{-1}\right)$ are the loading of dye and equilibrium adsorption capacity; $Q_{\mathrm{m}}\left(\mathrm{mg} \mathrm{g}^{-1}\right)$ is the maximum adsorption capacity; $K_{\mathrm{L}}\left(\mathrm{L} \mathrm{mg}^{-1}\right)$ is the Langmuir constant.
Another important parameter $R_{\mathrm{L}}$, the separation factor or equilibrium parameters, has been used to further estimate whether the Langmuir isothermal model fits the adsorption system. This factor can be calculated from the following equation:

Table 2 Kinetic parameters and correlation coefficients of dyes adsorption onto Ce-doped UiO-67

\begin{tabular}{|c|c|c|c|c|c|c|c|}
\hline \multirow[b]{2}{*}{ Organic dyes } & \multirow[b]{2}{*}{$Q_{\exp }\left(m g g^{-1}\right)$} & \multicolumn{3}{|c|}{ Pseudo-first-order model } & \multicolumn{3}{|c|}{ Pseudo-second-order model } \\
\hline & & $Q_{\mathrm{e}}\left(\mathrm{mg} \mathrm{g}^{-1}\right)$ & $K_{1}\left(\min ^{-1}\right)$ & $R^{2}$ & $Q_{\mathrm{e}}\left(\mathrm{mg} \mathrm{g}^{-1}\right)$ & $K_{2}\left(\min ^{-1}\right)$ & $R^{2}$ \\
\hline RhB & 754.4 & 164.6 & -0.00339 & 0.9423 & 747.6 & 0.00132 & 0.9994 \\
\hline MG & 589.2 & 119.9 & -0.16946 & 0.9345 & 583.1 & 0.00169 & 0.9992 \\
\hline
\end{tabular}



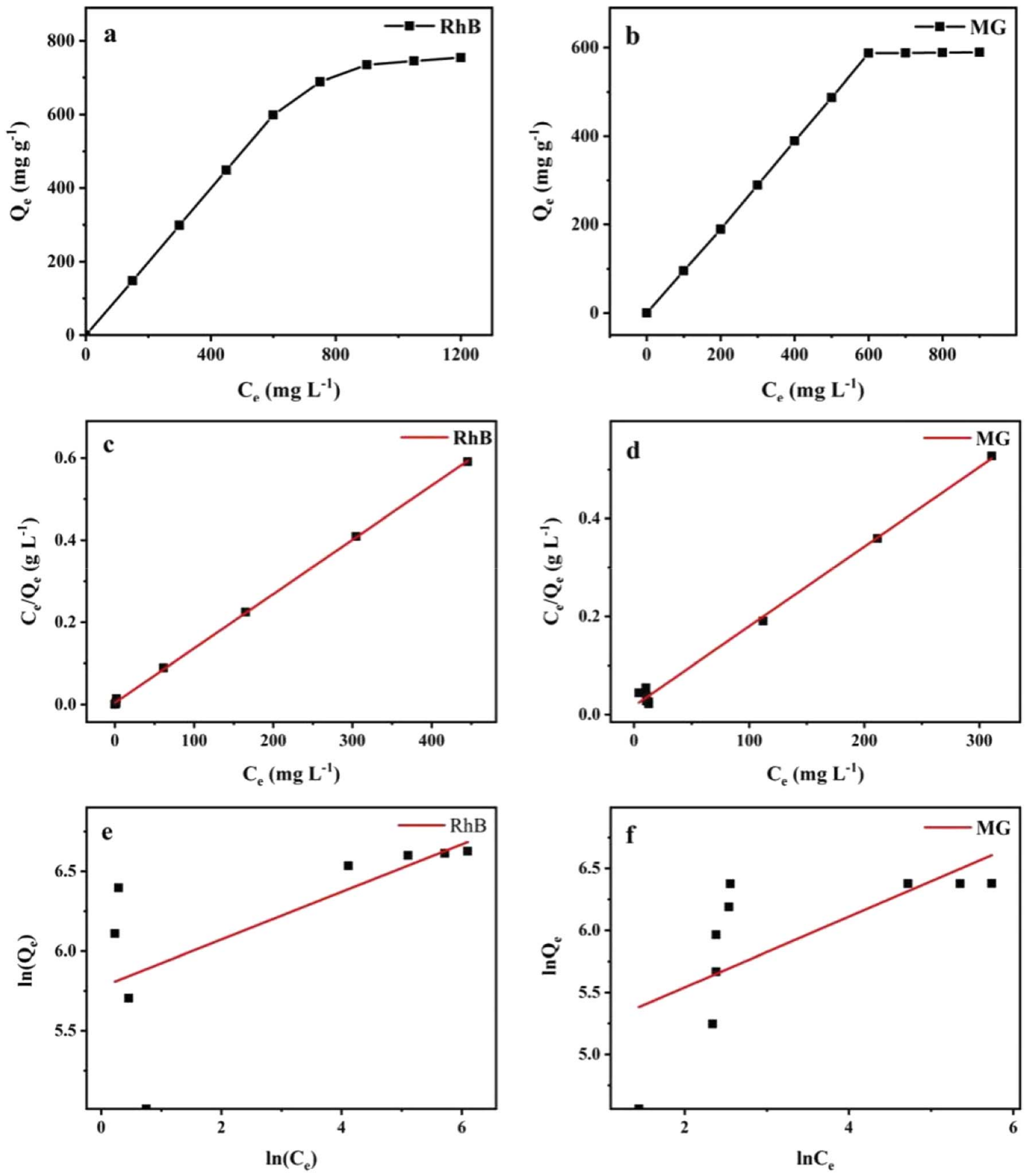

Fig. 7 (a and b) Adsorption isotherms of RhB and MG adsorbed by Ce-doped UiO-67; (c and d) Langmuir isotherm plot; (e and f) Freundlich isotherm plot.

$$
R_{\mathrm{L}}=1 /\left(1+K_{\mathrm{L}} C_{0}\right)
$$

where $C_{0}\left(\mathrm{mg} \mathrm{L}^{-1}\right)$ is the initial concentration of dye. The value of $R_{\mathrm{L}}$ can indicate that the isotherm type is irreversible $\left(R_{\mathrm{L}}=0\right)$, favorable $\left(0<R_{\mathrm{L}}<1\right)$, or unfavorable $\left(R_{\mathrm{L}}>1\right)$.

The Freundlich isothermal is expressed as follows:

$$
\ln Q_{\mathrm{e}}=\ln C_{\mathrm{e}} / n+\ln K_{\mathrm{F}}
$$

where $K_{\mathrm{F}}$ and $n$ are the Freundlich constants which involve the effect of initial dye concentration on adsorption.
From the fitting curves of Langmuir (Fig. 7(c)) and Freundlich kinetic models (Fig. 7(d)), the corresponding adsorption parameters are obtained (Table 4 ). The correlation coefficient $R^{2}$ of the Langmuir equation is close to unity and much larger than Freundlich model for both RhB and MG, indicating that the adsorption follows the Langmuir model. Moreover, the maximum adsorption capacities of RhB and MG calculated with the Langmuir model was considerably closer to experimental data. The $R_{\mathrm{L}}$ values of RhB and MG were great than 0 and far below 1, suggesting that adsorption for the dyes are favorable. 
Table 3 Comparison of cationic dyes adsorption onto different adsorbents

\begin{tabular}{|c|c|c|c|c|c|}
\hline \multirow{2}{*}{$\begin{array}{l}\text { Adsorbents } \\
\text { POM@UiO-66 }\end{array}$} & \multicolumn{4}{|c|}{ Adsorption capacity to cationic dyes $\left(\mathrm{mg} \mathrm{g}^{-1}\right)$} & \multirow{2}{*}{$\begin{array}{l}\text { Ref. } \\
42\end{array}$} \\
\hline & Rhodamine B & 222.6 & Malachite green & 190.6 & \\
\hline $\mathrm{UiO}-66-\mathrm{NH}_{2}$ & Methylene blue & 96.45 & - & - & 45 \\
\hline Zn-MOF & Rhodamine B & 2.977 & Methylene blue & 6.394 & 47 \\
\hline PCN-222 & Methylene blue & 589 & - & - & 48 \\
\hline Sulfonate lignin-based hydrogels (SLG) & Methylene blue & 495 & - & - & 49 \\
\hline Ce-doped UiO-67 & Rhodamine B & 754.4 & Malachite green & 589.2 & Current work \\
\hline
\end{tabular}

Table 4 Adsorption parameters of dyes in the Ce-doped UiO-67

\begin{tabular}{|c|c|c|c|c|c|c|c|c|}
\hline Organic dyes & $Q_{\exp }\left(\mathrm{mg} \mathrm{g}^{-1}\right)$ & $Q_{\mathrm{m}}\left(\mathrm{mg} \mathrm{g}^{-1}\right)$ & $K_{\mathrm{L}}\left(\mathrm{L} \mathrm{mg}^{-1}\right)$ & $R^{2}$ & $R_{\mathrm{L}}$ & $K_{\mathrm{F}}$ & $n$ & $R^{2}$ \\
\hline MG & 589.2 & 617.3 & 0.0919 & 0.9941 & 0.0981 & 144.2 & 3.5082 & 0.4071 \\
\hline
\end{tabular}

3.2.3. Effect of initial $\mathbf{p H}$. The solution $\mathrm{pH}$ is a factor for the adsorption process and influences the adsorption capacities of the adsorbents. Hence, the adsorption performance of MG onto Ce-doped UiO-67 was studied in the $\mathrm{pH}$ range of 3-10, and the results were depicted in Fig. 8. From the zeta potential measurements of Ce-doped UiO-67 (Fig. 8(b)), a zpc (point of zero charge) was observed at a $\mathrm{pH}$ of 5.05 , and the negative charge on the surface of the Ce-doped UiO-67 increases with rising $\mathrm{pH}$ of the $\mathrm{MG}$ solution in the $\mathrm{pH}$ range of 3.2-7. Since $\mathrm{MG}$ is a cationic dye, increasing the $\mathrm{pH}$ of the solution from 3.2 to 5 , favoured the electrostatic interaction between dye molecules and the adsorbent and thereby resulted into increased adsorption capacity of the material. Whereas, when the solution $\mathrm{pH}$ value above 3.5 , discoloration or precipitation will easily occur as shown in Fig. 9, due to MG is a triarylmethane conjugated basic dye and sensitive to $\mathrm{pH}$. Therefore, the optimal $\mathrm{pH}$ value for adsorption of MG occurs at 5 .
3.2.4. Selective adsorption ability. Selective adsorption ability is a crucial factor for application. We, therefore, used two cationic dyes (RhB and MG) and one anionic dye MO to see the result. The adsorption capacity for anionic dye MO (191.6 $\mathrm{mg} \mathrm{g}^{-1}$ ) is lower than those for $\mathrm{RhB}$ and MG. The results indicate that Ce doping changes the electrical property of UiO-67. Ce substitution to $\mathrm{Zr}$ takes place in the Ce-doped UiO-67, moreover the valence of $\mathrm{Zr}$ is +4 and the valence of $\mathrm{Ce}$ is +3 , thus Ce doping increases the negative charge on the surface of the material. And zeta potential measurements (Fig. 8(b)) reveal that the positive charge on the surface of the Ce-doped UiO-67 is much smaller than that of the UiO-67 in the $\mathrm{pH}$ range of 3.2-5.8. The electrostatic interactions between the adsorbent and the dye molecules determine the dye adsorption. The negative charge on the surface of Ce-doped UiO-67 structure within the $\mathrm{pH}$ range reduces the interactions with anionic dyes.
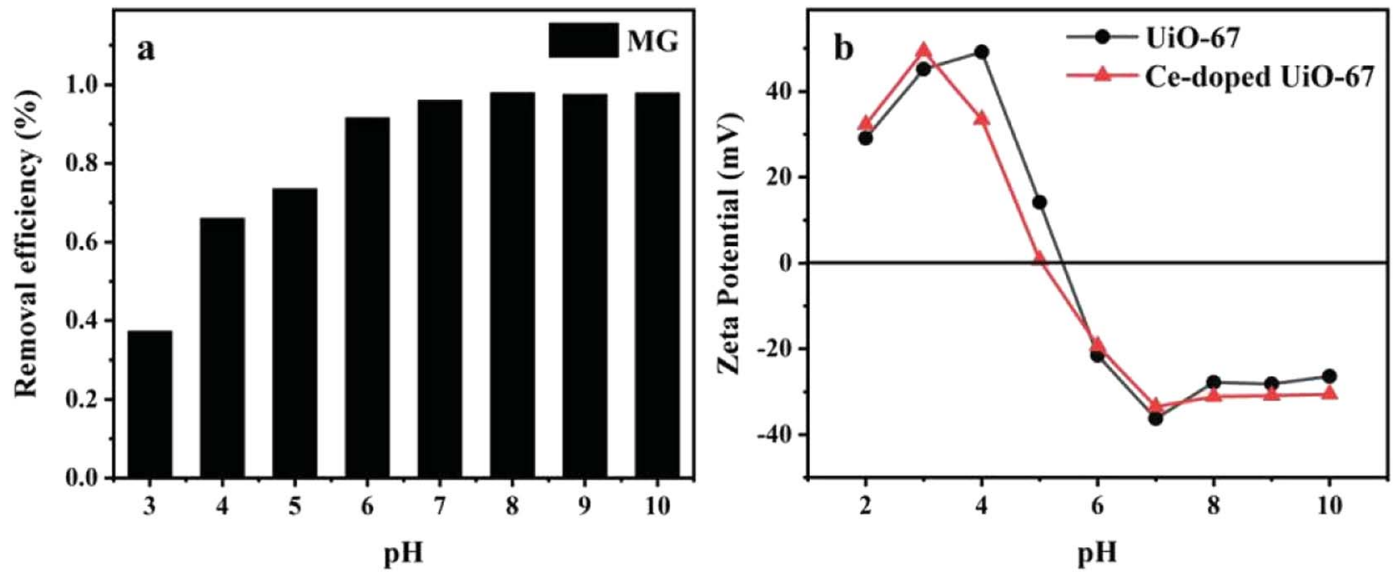

Fig. 8 (a) Effect of initial pH on adsorption capability of RhB. (b) Zeta potentials of UiO-67 and Ce-doped UiO-67 at various pH values. 
<smiles>CN(C)c1ccc(C(=C2C=CC(=[N+](C)C)C=C2)c2ccccc2)cc1</smiles>

Fig. 9 MG reaction equation.
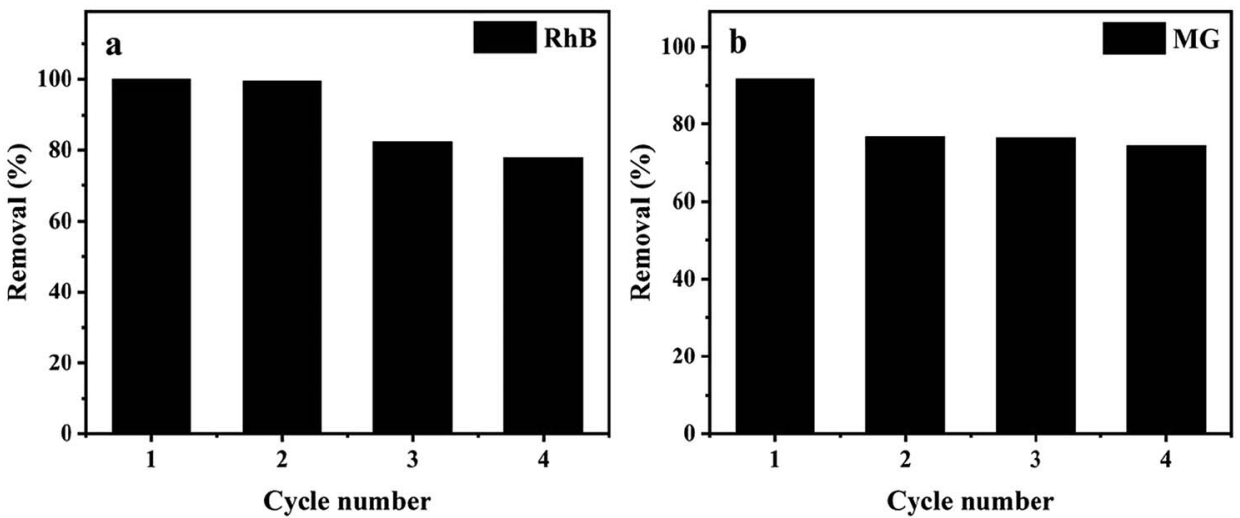

Fig. 10 (a) Recyclability of Ce-doped UiO-67 for the reduction of RhB and (b) recyclability of Ce-doped UiO-67 for the reduction of MG.

3.2.5. Reusability of the Ce-doped UiO-67. The reusability of an adsorbent is an important aspect considering applications. Thus, the reusability of Ce-doped UiO-67 was conducted by running repetitive adsorption cycles. The dye saturated Cedoped UiO-67 was added to ethylene glycol and ultrasonically treated. The separated out solid was washed with ethanol several times followed by drying under vacuum. Next cycle of adsorption was repeated. Ce-doped UiO-67 exhibits excellent reusability where both dyes removal reaches $75 \%$ after four time adsorption (Fig. 10), indicating good regeneration capacity and reusability.

\section{Conclusions}

In summary, we synthesized Ce-doped UiO-67 nanoparticles via a one-pot method. Ce doping increases electrostatic attraction between the adsorbent and the cationic dye molecules. As a result, the Ce-doped UiO-67 exhibited adsorption capacities of RhB (754.4 $\mathrm{mg} \mathrm{g}^{-1}$ ) and MG (589.2 $\mathrm{mg} \mathrm{g}^{-1}$ ) respectively, being significantly higher than those previously reported for UiO-67, RhB (41.3 $\mathrm{mg} \mathrm{g}^{-1}$ ) and $\mathrm{MG}$ (357.3 $\mathrm{mg} \mathrm{g}^{-1}$ ). Adsorption kinetics studies show that the adsorption process follows the pseudo-second-order kinetic model. The experiment data are well described by the Langmuir model. The Ce-doped UiO-67 after chemisorption can be regenerated and still has comparatively high adsorption capacity after four time adsorptiondesorption cycles. Its adsorption efficiency and simple synthesis process warrant the potential use of the Ce-doped UiO-67 material as an adsorbent for wastewater treatment.

\section{Conflicts of interest}

There are no conflicts to declare.

\section{References}

1 M. T. Yagub, T. K. Sen, S. Afroze and H. M. Ang, Adv. Colloid Interface Sci., 2014, 209, 172-184.

2 A. K. Verma, R. R. Dash and P. Bhunia, J. Environ. Manage., 2012, 93, 154-168.

3 G. Boczkaj and A. Fernandes, Chem. Eng. J., 2017, 320, 608633.

4 Z. Shi, L. Li, Y. Xiao, Y. Wang, K. Sun, H. Wang and L. Liu, RSC Adv., 2017, 7, 30904-30910.

5 H. Wei, B. Gao, J. Ren, A. Li and H. Yang, Water Res., 2018, 143, 608-631. 
6 J. Zhang, Z. Xiong and X. S. Zhao, J. Mater. Chem., 2011, 21, 3634.

7 X. J. Li, H. J. Li, M. J. Li, C. P. Li, D. Z. Sun, Y. J. Lei and B. H. Yang, Carbon, 2018, 129, 543-551.

8 I. Oller, S. Malato and J. A. Sanchez-Perez, Sci. Total Environ., 2011, 409, 4141-4166.

9 Y. Oh, D. L. Armstrong, C. Finnerty, S. X. Zheng, M. Hu, A. Torrents and B. X. Mi, J. Membr. Sci., 2017, 541, 235-243.

10 S. Shanmuganathan, P. Loganathan, C. Kazner, M. A. H. Johir and S. Vigneswaran, Desalination, 2017, 401, 134-141.

11 Y. Q. Zhan, X. Y. Wan, S. J. He, Q. B. Yang and Y. He, Chem. Eng. J., 2018, 333, 132-145.

12 J. Wang, Y. Han, H. Xu and Z.-L. Xu, Appl. Organomet. Chem., 2018, 32, e4097.

13 X. Zhao, Y. Wang, D. S. Li, X. Bu and P. Feng, Adv. Mater., 2018, 30, e1705189.

14 N. Ko, J. Hong, S. Sung, K. E. Cordova, H. J. Park, J. K. Yang and J. Kim, Dalton Trans., 2015, 44, 2047-2051.

15 J. Duan, S. Chen and C. Zhao, Nat. Commun., 2017, 8, 15341.

16 F.-h. Wei, D. Chen, Z. Liang, S.-q. Zhao and Y. Luo, RSC Adv., 2017, 7, 46520-46528.

17 B. An, J. Zhang, K. Cheng, P. Ji, C. Wang and W. Lin, J. Am. Chem. Soc., 2017, 139, 3834-3840.

18 I. Abánades Lázaro and R. S. Forgan, Coord. Chem. Rev., 2019, 380, 230-259.

19 Y. Li, Y. Su, J. Xu, Z.-l. Xu and H. Xu, Bull. Chem. Soc. Jpn., 2017, 90, 1152-1156.

20 A. H. Assen, O. Yassine, O. Shekhah, M. Eddaoudi and K. N. Salama, ACS Sens., 2017, 2, 1294-1301.

21 R. Abazari, A. R. Mahjoub and J. Shariati, J. Hazard. Mater., 2019, 366, 439-451.

22 R. Balderas-Xicohtencatl, M. Schlichtenmayer and M. Hirscher, Energy Technol., 2018, 6, 578-582.

23 M. J. Katz, Z. J. Brown, Y. J. Colon, P. W. Siu, K. A. Scheidt, R. Q. Snurr, J. T. Hupp and O. K. Farha, Chem. Commun., 2013, 49, 9449-9451.

24 H. Xu, X. Luo, J. Wang, Y. Su, X. Zhao and Y. Li, ACS Appl. Mater. Interfaces, 2019, 11, 20291-20297.

25 H. Xu, Y. Li, X. Luo, Z. Xu and J. Ge, Chem. Commun., 2017, 53, 7953-7956.

26 J. M. Yang, Q. Liu and W. Y. Sun, Microporous Mesoporous Mater., 2014, 190, 26-31.

27 L. N. Li, S. S. Shen, W. P. Ai, S. Y. Song, Y. Bai and H. W. Liu, Sens. Actuators, B, 2018, 267, 542-548.

28 J. Cao, Z.-h. Yang, W.-p. Xiong, Y.-y. Zhou, Y.-r. Peng, X. Li, C.-y. Zhou, R. Xu and Y.-r. Zhang, Chem. Eng. J., 2018, 353, 126-137.
29 X. Yang, S. Deng, F. Peng and T. Luo, Dalton Trans., 2017, 46, 1996-2006.

30 M. Wu, X. Hu, C. Li, J. Li, H. Zhou, X. Zhang and R. Liu, Int. J. Hydrogen Energy, 2018, 43, 14701-14709.

31 M. R. D. Khaki, M. S. Shafeeyan, A. A. A. Raman and W. Daud, J. Environ. Manage., 2017, 198, 78-94.

32 L. B. Jiang, X. Z. Yuan, Y. Pan, J. Liang, G. M. Zeng, Z. B. Wu and H. Wang, Appl. Catal., B, 2017, 217, 388-406.

33 E. Žunkovič, M. Mazaj, G. Mali, M. Rangus, T. Devic, C. Serre and N. Z. Logar, J. Solid State Chem., 2015, 225, 209-215.

34 Q. F. Yang, J. Wang, X. Y. Chen, W. X. Yang, H. N. Pei, N. Hu, Z. H. Li, Y. R. Suo, T. Li and J. L. Wang, J. Mater. Chem. A, 2018, 6, 2184-2192.

35 Q. Yang, Y. Wang, J. Wang, F. Liu, N. Hu, H. Pei, W. Yang, Z. Li, Y. Suo and J. Wang, Food Chem., 2018, 254, 241-248.

36 H. Duo, Y. Wang, L. Wang, X. Lu and X. Liang, J. Sep. Sci., 2018, 41, 4149-4158.

37 Z. W. Yang, X. Q. Xu, X. X. Liang, C. Lei, L. H. Gao, R. X. Hao, D. D. Lu and Z. Q. Lei, Appl. Surf. Sci., 2017, 420, 276-285.

38 A. M. Ebrahim and T. J. Bandosz, ACS Appl. Mater. Interfaces, 2013, 5, 10565-10573.

39 X. Zhu, B. Li, J. Yang, Y. Li, W. Zhao, J. Shi and J. Gu, ACS Appl. Mater. Interfaces, 2015, 7, 223-231.

40 S. C. Liu, J. Xu, E. G. Dai, J. J. Qiu and Y. Liu, Microporous Mesoporous Mater., 2018, 264, 133-138.

41 Q. Liang, S. Cui, C. Liu, S. Xu, C. Yao and Z. Li, J. Colloid Interface Sci., 2018, 524, 379-387.

42 L. Zeng, L. Xiao, Y. Long and X. Shi, J. Colloid Interface Sci., 2018, 516, 274-283.

43 J. M. Yang, J. Colloid Interface Sci., 2017, 505, 178-185.

44 H. Ali-Moussa, R. Navarro Amador, J. Martinez, F. Lamaty, M. Carboni and X. Bantreil, Mater. Lett., 2017, 197, 171-174.

45 Q. Chen, Q. Q. He, M. M. Lv, Y. L. Xu, H. B. Yang, X. T. Liu and F. Y. Wei, Appl. Surf. Sci., 2015, 327, 77-85.

46 P. Hu, Z. Zhao, X. Sun, Y. Muhammad, J. Li, S. Chen, C. Pang, T. Liao and Z. Zhao, Chem. Eng. J., 2019, 356, 329-340.

47 J. Zhang, F. Li and Q. Sun, Appl. Surf. Sci., 2018, 440, 12191226.

48 H. Li, X. Cao, C. Zhang, Q. Yu, Z. Zhao, X. Niu, X. Sun, Y. Liu, L. Ma and Z. Li, RSC Adv., 2017, 7, 16273-16281.

49 J. Li, H. Li, Z. Yuan, J. Fang, L. Chang, H. Zhang and C. Li, Int. J. Biol. Macromol., 2019, 135, 1171-1181.

50 Y. Zhang, K. Xia, X. Liu, Z. Chen, H. Du and X. Zhang, J. Taiwan Inst. Chem. Eng., 2019, 102, 1-8.

51 Z. Liang, Z. Zhao, T. Sun, W. Shi and F. Cui, J. Colloid Interface Sci., 2017, 485, 192-200. 